\title{
CHICOM: A code of tests for comparing unweighted and weighted histograms and two weighted histograms
}

\author{
N.D. Gagunashvilia,* \\ ${ }^{a}$ University of Akureyri, Borgir, v/Nordurslód, IS-600 Akureyri, Iceland
}

\begin{abstract}
A Fortran-77 program for calculating test statistics to compare weighted histogram with an unweighted histogram and two histograms with weighted entries is presented. The code calculates test statistics for cases of histograms with normalized weights of events and unnormalized weights of events.

Keywords: homogeneity test, fit Monte Carlo distribution to data, comparison experimental and simulated data, data interpretation
\end{abstract}

\section{PROGRAM SUMMARY}

\section{Program Title: CHICOM}

Journal Reference:

Catalogue identifier:

Licensing provisions: none

Programming language: Fortran-77

Computer: Any Unix/Linux workstation or PC with a Fortran-77 compiler.

Classification: $4.13,11.9,16.4,19.4$

External routines/libraries used: FPLSOR (M103) [1] and BRENT [2]

Nature of problem: The program calculates test statistics for comparing two weighted histograms and an unweighted histogram with a weighted one.

Solution method: Calculation of test statistics is done according formulas presented in Ref. [3].

Running time: $0.001 \mathrm{sec}$ for 5 bins histogram.

\footnotetext{
${ }^{*}$ Corresponding author.

E-mail address: nikolai@simnet.is
} 


\section{References}

[1] CERN Program Library, http://cernlib.web.cern.ch/cernlib/.

[2] W.H. Press, S.A. Teukolsky, W.T. Vetterling, B.P. Flannery, Numerical Recipes in Fortran 77 the Art of Scientific Computing, Second Edition, Cambridge University Press, 1997.

[3] N.D. Gagunashvili, Nucl. Instr. Meth. A614 (2010) 287-296.

\section{Introduction}

A histogram with $m$ bins for a given probability density function $p(x)$ is used to estimate the probabilities $p_{i}$ that a random event belongs in bin $i$ :

$$
p_{i}=\int_{S_{i}} p(x) d x, i=1, \ldots, m .
$$

Integration in (11) is carried out over the bin $S_{i}$ and $\sum_{1}^{m} p_{i}=1$. A histogram can be obtained as a result of a random experiment with the probability density function $p(x)$.

A frequently used technique in data analysis is the comparison of two distributions through the comparison of histograms. The hypothesis of homogeneity [1] is that the two histograms represent random values with identical distributions. It is equivalent to there existing $m$ constants $p_{1}, \ldots, p_{m}$, such that $\sum_{i=1}^{m} p_{i}=1$, and the probability of belonging to the $i$ th bin for some measured value in both experiments is equal to $p_{i}$.

Let us denote the number of random events belonging to the $i$ th bin of the first and second histograms as $n_{1 i}$ and $n_{2 i}$, respectively. The total number of events in the histograms are equal to $n_{j}=\sum_{i=1}^{m} n_{j i}$, where $j=1,2$.

As shown in [1] the statistic

$$
\frac{1}{n_{1} n_{2}} \sum_{i=1}^{m} \frac{\left(n_{2} n_{1 i}-n_{1} n_{2 i}\right)^{2}}{n_{1 i}+n_{2 i}}
$$

has approximately a $\chi_{m-1}^{2}$ distribution if hypothesis of homogeneity is valid.

Weighted histograms are often obtained as a result of Monte-Carlo simulations. References [2, 3, 4] are examples of research on high-energy physics, statistical mechanics, and astrophysics using such histograms. 
To define a weighted histogram let us write the probability $p_{i}$ (1) for a given probability density function $p(x)$ in the form

$$
p_{i}=\int_{S_{i}} p(x) d x=\int_{S_{i}} w(x) g(x) d x,
$$

where

$$
w(x)=p(x) / g(x)
$$

is the weight function and $g(x)$ is some other probability density function. The function $g(x)$ must be $>0$ for points $x$, where $p(x) \neq 0$. The weight $w(x)=0$ if $p(x)=0$, see Ref. [5]. Because of the condition $\sum_{i} p_{i}=1$ further we will call the above defined weights normalized weights as opposed to the unnormalized weights $\breve{w}(x)$ which are $\check{w}(x)=$ const $\cdot w(x)$.

The histogram with normalized weights was obtained from a random experiment with a probability density function $g(x)$, and the weights of the events were calculated according to (4). Let us denote the total sum of the weights of the events in the $i$ th bin of the histogram with normalized weights as

$$
W_{i}=\sum_{l=1}^{n_{i}} w_{i}(l),
$$

where $n_{i}$ is the number of events at bin $i$ and $w_{i}(l)$ is the weight of the $l$ th event in the $i$ th bin. The total number of events in the histogram is equal to $n=\sum_{i=1}^{m} n_{i}$, where $m$ is the number of bins. The quantity $\hat{p}_{i}=W_{i} / n$ is the estimator of $p_{i}$ with the expectation value $E\left[\hat{p}_{i}\right]=p_{i}$. Note that in the case where $g(x)=p(x)$, the weights of the events are equal to 1 and the histogram with normalized weights is the usual histogram with unweighted entries.

Let us introduce notations need for the description of tests for comparing histograms:

- $W_{j i}=\sum_{l=1}^{n_{j i}} w_{j i}(l)$ - the total sum of the weights of the events in the $i$ th bin of the $j$ th the histogram with normalized weights;

- $r_{j i}=\sum_{l=1}^{n_{j i}} w_{j i}(l) / \sum_{l=1}^{n_{j i}} w_{j i}^{2}(l)$ - estimator of the ratio of moments in the $i$ th bin of the $j$ th histogram with normalized weights. 
And the same quantities we introduce for the histograms with unnormalized weighted entries:

- $\check{W}_{j i}=\sum_{l=1}^{n_{2 i}} \check{w}_{j i}(l)$

- $\check{r}_{j i}=\sum_{l=1}^{n_{j i}} \check{w}_{j i}(l) / \sum_{l=1}^{n_{j i}} \check{w}_{j i}^{2}(l)$

Notice that $W_{j i}=n_{j i}$ and $r_{j i}=1$ for histograms with unweighted entries.

Three types of statistics used for comparing histograms are presented at Ref [6].

\section{Histograms with normalized weighted entries.}

Let us introduce the statistic

$$
{ }_{1} X_{k}^{2}=\sum_{j=1}^{2} \frac{1}{n_{j}} \sum_{i \neq k} \frac{r_{j i} W_{j i}^{2}}{p_{i}}+\sum_{j=1}^{2} \frac{1}{n_{j}} \frac{\left(n_{j}-\sum_{i \neq k} r_{j i} W_{j i}\right)^{2}}{1-\sum_{i \neq k} r_{j i} p_{i}}-\sum_{j=1}^{2} n_{j} .
$$

with the sums in (6) extending over all bins $i$ except one bin $k$. In the equation (6), the probabilities $p_{i}$ are unknown, and estimators $\hat{p}_{i}$ of the probabilities are found by minimization of (6) $)$. We denote by ${ }_{1} \hat{X}_{k}^{2}$ the value of ${ }_{1} X_{k}^{2}$ after substitution of the estimators $\hat{p}_{i}$ into (6). As shown in [6], the statistic

$$
{ }_{1} X^{2}=\operatorname{Med}\left\{{ }_{1} \hat{X}_{1}^{2},{ }_{1} \hat{X}_{2}^{2}, \ldots,{ }_{1} \hat{X}_{m}^{2}\right\}
$$

approximately has a $\chi_{m-1}^{2}$ distribution if the hypothesis of homogeneity is valid.

\section{Histograms with unnormalized weighted entries.}

Let us introduce the statistic

$$
{ }_{2} X_{k}^{2}=\sum_{j=1}^{2} \frac{s_{k j}^{2}}{n_{j}}+2 \sum_{j=1}^{2} s_{k j},
$$

where

$$
s_{k j}=\sqrt{\sum_{i \neq k} \check{r}_{j i} p_{i} \sum_{i \neq k} \check{r}_{j i} \check{W}_{j i}^{2} / p_{i}}-\sum_{i \neq k} \check{r}_{j i} \check{W}_{j i} .
$$

Again estimators $\hat{p}_{i}$ of unknown probabilities $p_{i}$ are found by minimization of (8). We denote by ${ }_{2} \hat{X}_{k}^{2}$ the value of ${ }_{2} X_{k}^{2}$ after substitution of the estimators $\hat{p}_{i}$ into (8). As shown in [6], the statistic

$$
{ }_{2} X^{2}=\operatorname{Med}\left\{{ }_{2} \hat{X}_{1}^{2},{ }_{2} \hat{X}_{2}^{2}, \ldots,{ }_{2} \hat{X}_{m}^{2}\right\}
$$


approximately has a $\chi_{m-2}^{2}$ distribution if the hypothesis of homogeneity is valid.

Histograms with normalized and unnormalized weighted entries.

Let us introduce the statistic

$$
{ }_{3} X_{k}^{2}=\frac{1}{n_{1}} \sum_{i \neq k} \frac{r_{1 i} W_{1 i}^{2}}{p_{i}}+\frac{1}{n_{1}} \frac{\left(n_{1}-\sum_{i \neq k} r_{1 i} W_{1 i}\right)^{2}}{1-\sum_{i \neq k} r_{1 i} p_{i}}-n_{1}+\frac{s_{k 2}^{2}}{n_{2}}+2 s_{k 2} .
$$

We denote by ${ }_{3} \hat{X}_{k}^{2}$ the value of ${ }_{3} X_{k}^{2}$ after substitution of the estimators $\hat{p}_{i}$ into (11). As shown in [6], the statistic

$$
{ }_{3} X^{2}=\operatorname{Med}\left\{{ }_{3} \hat{X}_{1}^{2},{ }_{3} \hat{X}_{2}^{2}, \ldots,{ }_{3} \hat{X}_{m}^{2}\right\}
$$

approximately has a $\chi_{m-2}^{2}$ distribution if the hypothesis of homogeneity is valid.

The chi-square approximation is asymptotic. This means that the critical values may not be valid if the expected frequencies are too small. The use of the chi-square test is inappropriate if any expected frequency is $<1$, or if the expected frequency is $<5$ in $>20 \%$ of the bins for either histogram. This restriction observed in the usual chi-square test [7] is quite reasonable for the proposed test.

Information for readers. Recently, another paper dedicated to weighted histograms has been published in "Computer Physics Communication", see Ref. [9]. The same author has presented a program for goodness of fit test for histograms with weighted and unweighted entries. The test is used in a data analysis for comparison theoretical frequencies with frequencies represented by histogram.

\section{Computer program}

CHICOM is a subroutine which can be called from the Fortran programs for calculating test statistics ${ }_{1} X^{2},{ }_{2} X^{2}$ and ${ }_{3} X^{2}$.

\section{Usage}

CALL CHICOM (AEX , ERAEX , NEV , AMC , ERAMC, NMC , NCHA , MODE , STAT , NDF , IFAIL)

Input Data 
AEX - one dimensional real array of first weighted histogram content

ERAEX - one dimensional real array of histogram content for entries of first histogram with squares of weights.

$\mathrm{NEV}$ - number of events in the first histogram $n_{1}$

AMC - one dimensional real array of second weighted histogram content

ERAMC - one dimensional real array of histogram content for entries of second histogram with squares of weights.

$\mathrm{NMC}$ - number of events in the second histogram $n_{2}$

NCHA - number of bins $m$

MODE - equal 1 for both histograms with normalized weights, equal 2 for both histograms with unnormalized weights equal 3 for first histogram with normalized weights and the second with unnormalized weights

Output data

STAT - test statistic

$\mathrm{NDF}$ - number of degree of freedom $l$ of the $\chi_{l}^{2}$ distribution if hypothesis $H_{0}$ is true (will be $l=m-1$ or $l=m-2$ )

IFAIL - will be $>0$ if calculation is not successful.

\section{Test run}

We take a distribution:

$$
p(x) \propto \frac{2}{(x-10)^{2}+1}+\frac{1}{(x-14)^{2}+1}
$$

defined on the interval $[4,16]$ and representing two so-called Breit-Wigner peaks [8]. Three cases of the probability density function $g(x)$ are considered 


$$
\begin{gathered}
g_{1}(x)=p(x) \\
g_{2}(x)=1 / 12 \\
g_{3}(x) \propto \frac{2}{(x-9)^{2}+1}+\frac{2}{(x-15)^{2}+1}
\end{gathered}
$$

Distribution $g_{1}(x)$ (14) results in a histogram with unweighted entries, while distribution $g_{2}(x)(15)$ is a uniform distribution on the interval $[4,16]$. Distribution $g_{3}(x)$ (16) has the same form of parametrization as $p(x)$ (13), but with different values for the parameters.

Three cases were considered:

\begin{tabular}{|l|l|l|l|l|}
\hline & \multicolumn{2}{|l|}{ First histogram } & \multicolumn{2}{l|}{ Second histogram } \\
\hline № & type of weight & weight & type of weight & weight \\
\hline 1 & normalized & $p(x) / g_{1}(x)=1$ & normalized & $p(x) / g_{1}(x)=1$ \\
2 & unnormalized & $0.5 p(x) / g_{2}(x)$ & unnormalized & $2 p(x) / g_{3}(x)$ \\
3 & normalized & $p(x) / g_{1}(x)=1$ & unnormalized & $0.5 p(x) / g_{3}(x)$ \\
\hline
\end{tabular}

For each case histograms with 5 bins were created by simulation 500 entries for first histogram and 1000 entries for the second one. The results of the calculations are presented below.

\section{Test 1}

\section{INPUT}

$\begin{array}{lccccc}\text { AEX } & 11.0000 & 58.0000 & 234.0000 & 102.0000 & 95.0000 \\ \text { ERAEX } & 11.0000 & 58.0000 & 234.0000 & 102.0000 & 95.0000 \\ \text { NEV } & 500 & & & & \\ \text { AMC } & 30.0000 & 119.0000 & 439.0000 & 182.0000 & 230.0000 \\ \text { ERAMC } & 30.0000 & 119.0000 & 439.0000 & 182.0000 & 230.0000 \\ \text { NMC } & 1000 & & & & \\ \text { NCHA } & 5 & & & & \\ \text { MODE } & 1 & & & & \end{array}$


OUTPUT

$\begin{array}{lll}\text { STAT } & 4.7391 & (\mathrm{p} \text {-value }=0.3151) \\ \text { NDF } & 4 & \\ \text { IFAIL } & 0 & \end{array}$

\section{Test 2}

INPUT

\begin{tabular}{lrrrrr} 
AEX & 9.3018 & 22.8871 & 122.0670 & 51.6786 & 46.2622 \\
ERAEX & 0.8026 & 7.7173 & 142.7876 & 27.7087 & 28.5724 \\
NEV & 500 & & & & \\
AMC & 68.9455 & 213.5029 & 898.8528 & 397.7258 & 419.0171 \\
ERAMC & 108.3022 & 229.3163 & 3697.7102 & 1455.0262 & 699.6888 \\
NMC & 1000 & & & & \\
NCHA & 5 & & & & \\
MODE & 2 & & & & \\
& & & & & \\
& \multicolumn{2}{c}{ OUTPUT } & & & \\
& & & & & \\
STAT & 1.9111 & & & & \\
NDF & 3 & & & & \\
IFAIL & 0 & & & &
\end{tabular}

Test 3

INPUT

$\begin{array}{lrrrrr}\text { AEX } & 17.0000 & 53.0000 & 225.0000 & 101.0000 & 104.0000 \\ \text { ERAEX } & 17.0000 & 53.0000 & 225.0000 & 101.0000 & 104.0000 \\ \text { NEV } & 500 & & & & \\ \text { AMC } & 14.2303 & 53.9921 & 204.9794 & 111.6337 & 101.1128 \\ \text { ERAMC } & 5.4897 & 14.5935 & 198.6223 & 103.7259 & 40.9275 \\ \text { NMC } & 1000 & & & & \\ \text { NCHA } & 5 & & & & \\ \text { MODE } & 3 & & & & \end{array}$

OUTPUT 


$\begin{array}{lll}\text { STAT } & 1.4431 & (\mathrm{p} \text {-value }=0.6955) \\ \text { NDF } & 3 & \\ \text { IFAIL } & 0 & \end{array}$

\section{References}

[1] H. Cramer, Mathematical methods of statistics, Princeton University Press, Princeton, 1999.

[2] V.M. Abazov et al., D0 Collaboration, Phys. Lett. B693 (2010) 515-521.

[3] A.M. Ferrenberg and R. H. Swendsen, Phys. Rev. Lett. 61 (1988) 26352638.

[4] L.A. Pozdniakov, I.M. Sobol, R.A. Siuniaev, Comptonization and the shaping of X-ray source spectra - Monte Carlo calculations, in Soviet Scientific Reviews, Section E: Astrophysics and Space Physics Reviews, vol. 2, 189-331, Harwood Academic, New York, 1983.

[5] I. Sobol, A Primer For The Monte Carlo Method, CRC Press, Boca Raton, Florida, 1994.

[6] N.D. Gagunashvili, Nucl. Instr. Meth. A614 (2010) 287-296.

[7] D.S. Moore, G.P. McCabe, Introduction to the Practice of Statistics, W.H. Freeman Publishing Company, New York, 2005.

[8] G. Breit, E. Wigner, Phys. Rev. 49 (1936) 519-531.

[9] N.D. Gagunashvili, Comp. Phys. Comm. CPC-D-11-00117R1 